\title{
Search for brown-dwarf companions of stars (Corrigendum)
}

\author{
J. Sahlmann ${ }^{1,2}$, D. Ségransan ${ }^{1}$, D. Queloz ${ }^{1}$, S. Udry ${ }^{1}$, N. C. Santos ${ }^{3,4}$, M. Marmier ${ }^{1}$, M. Mayor ${ }^{1}$, \\ D. Naef ${ }^{1}$, F. Pepe ${ }^{1}$, and S. Zucker ${ }^{5}$ \\ 1 Observatoire de Genève, Université de Genève, 51 chemin Des Maillettes, 1290 Sauverny, Switzerland \\ e-mail: johannes.sahlmann@sciops.esa.int \\ 2 European Southern Observatory, Karl-Schwarzschild-Str. 2, 85748 Garching bei München, Germany \\ 3 Centro de Astrofísica, Universidade do Porto, Rua das Estrelas, 4150-762 Porto, Portugal \\ 4 Departamento de Física e Astronomia, Faculdade de Ciências, Universidade do Porto, 4169-007 Porto, Portugal \\ 5 Department of Geophysics and Planetary Sciences, Tel Aviv University, 69978 Tel Aviv, Israel
}

\section{A\&A 525, A95 (2011), DOI: 10.1051/0004-6361/201015427}

Key words. binaries: spectroscopic - brown dwarfs - stars: low-mass - techniques: radial velocities - astrometry - errata, addenda

In Table 1 of our original publication, the values for the HIPPARCos identifier, $V, B-V$, and the parallax of the two stars HD 53680 and HD 52756 were mixed up by mistake. None of the results in our paper are affected by this error.

Acknowledgements. We thank Arpita Roy of Pennsylvania State University who drew our attention to this mistake.
Table 1. HiPparcos parameters of the surveyed stars.

\begin{tabular}{rcccccc}
\hline \hline Nr. & \multicolumn{2}{c}{ Object } & Sp. T. & $V$ & $B-V$ & $\begin{array}{c}\varpi \\
\text { (mas) }\end{array}$ \\
\hline 1 & 3277 & 2790 & G8V & 7.59 & 0.73 & $34.7 \pm 0.7$ \\
2 & 4747 & 3850 & G8 & 7.30 & 0.77 & $53.5 \pm 0.5$ \\
3 & 17289 & 12726 & G0V & 7.56 & 0.59 & $20.8 \pm 1.2$ \\
4 & 30501 & 22122 & K0V & 7.73 & 0.88 & $47.9 \pm 0.5$ \\
5 & 43848 & 29804 & K1IV & 8.80 & 0.93 & $26.4 \pm 0.8$ \\
6 & 52756 & 33736 & K1V & 8.61 & 0.90 & $30.1 \pm 0.6$ \\
7 & 53680 & 34052 & K5V & 8.80 & 1.18 & $57.4 \pm 1.2$ \\
8 & 74014 & 42634 & K0 & 7.73 & 0.76 & $29.6 \pm 0.7$ \\
9 & 89707 & 50671 & G1V & 7.29 & 0.55 & $30.6 \pm 0.6$ \\
10 & 154697 & 83770 & G5 & 7.97 & 0.73 & $30.6 \pm 1.0$ \\
11 & 164427 A & 88531 & G4IV & 7.01 & 0.62 & $26.3 \pm 0.7$ \\
12 & 167665 & 89620 & F8V & 6.48 & 0.54 & $32.8 \pm 0.5$ \\
13 & 189310 & 99634 & K2V & 8.61 & 0.90 & $31.0 \pm 0.6$ \\
14 & 211847 & 110340 & G5V & 8.78 & 0.66 & $19.8 \pm 1.3$ \\
15 & $\cdots$ & 103019 & K5 & 10.45 & 1.33 & $28.3 \pm 2.3$ \\
\hline
\end{tabular}

